\title{
Exp-type Ulam-Hyers stability of fractional differential equations with positive constant coefficient
}

\author{
Zhuoyan Gao ${ }^{1}$, Xiulan Yu and JinRong Wang ${ }^{2 *}$
}

\section{"Correspondence:}

sci.jrwang@gzu.edu.cn

2Department of Mathematics,

Guizhou University, Guiyang,

Guizhou 550025, P.R. China

Full list of author information is

available at the end of the article

\begin{abstract}
In this paper, we apply asymptotic behavior on Mittag-Leffler functions $\mathbb{E}_{\alpha}(z)$ and $\mathbb{E}_{\alpha, \alpha}(z)$ for $z>0$ to discuss exp-type Ulam-Hyers stability of ${ }^{\mathrm{c}} D_{t}^{\alpha} x(t)=\lambda x(t)+f(t, x(t))$ for the case $\lambda>0$ on a finite time interval $[0,1]$ and an unbounded interval $(1, \infty)$.
\end{abstract}

MSC: exp-type Ulam-Hyers stability; fractional differential equations; asymptotic behavior

Keywords: 26A33; 34D10; 45N05

\section{Introduction}

Since SM Ulam raised the famous question on the stability of functional equations, more and more researchers generalize, improve, and extend Ulam's type stability problems of differential equations. For more contributions on such area, one can refer to [1-6] and the references therein.

Recently, the authors [7] discussed Ulam's type stability of fractional differential equations

$$
{ }^{\mathrm{c}} D_{t}^{\alpha} x(t)=\lambda x(t)+f(t, x(t)), \quad \alpha \in(0,1), t \in J, \lambda<0,
$$

on finite time interval $J$, where ${ }^{\mathrm{c}} D_{t}^{\alpha}$ is the Caputo fractional derivative of order $\alpha \in$ $(0,1)$ with the lower limit zero (see Definition 2.1). The main tools and techniques are based on the estimation on Mittag-Leffler functions $\mathbb{E}_{\alpha}(z):=\sum_{k=0}^{\infty} \frac{z^{k}}{\Gamma(k \alpha+1)}$ and $\mathbb{E}_{\alpha, \alpha}(z):=$ $\sum_{k=0}^{\infty} \frac{z^{k}}{\Gamma(\alpha k+\alpha)}$ for $z \leq 0$ (see Lemma 2 in [8]) and a singular Gronwall type integral inequality (see Theorem 1 in [9]).

The classical concept of Ulam-Hyers stability (see [10]) has applicable significance since it means that if we are dealing with Ulam-Hyers stable system then one does not seek the exact solution. All what is required is to find a function which satisfies a suitable approximation inequality. In other words, Ulam-Hyers stability can guarantee that there exists a close exact solution. This approach is quite useful in many applications such as numerical analysis and optimization, where seeking the exact solution is impossible.

In this paper, we are interested in discussing exp-type Ulam-Hyers stability (see Definition 1.1), a special Ulam-Hyers-Rassias stability, of equation (1) for the case $\lambda>0$, on a 
finite time interval $J=[0,1]$ and an unbounded interval $J=(1, \infty)$, i.e.,

$$
{ }^{\mathrm{c}} D_{t}^{\alpha} x(t)=\lambda x(t)+f(t, x(t)), \quad \alpha \in(0,1), t \in J, \lambda>0 .
$$

Let $\epsilon>0, f: J \times \mathbb{R} \rightarrow \mathbb{R}$ be a continuous function. Consider equation (2) and the following inequality:

$$
\left|{ }^{\mathrm{c}} D_{t}^{\alpha} y(t)-\lambda y(t)-f(t, y(t))\right| \leq \epsilon, \quad \alpha \in(0,1), t \in J, \lambda>0 .
$$

Definition 1.1 Let $C(J, \mathbb{R})$ be the set of all continuous functions from $J$ into $\mathbb{R}$. Equation (2) is exp-type Ulam-Hyers stable if there exists $c>0$ such that for each $\epsilon>0$ and for each solution $y \in C(J, \mathbb{R})$ of inequality (3) there exists a solution $x \in C(J, \mathbb{R})$ of equation (2) with

$$
|y(t)-x(t)| \leq c \epsilon e^{\lambda^{\frac{1}{\alpha}} t}, \quad \alpha \in(0,1), t \in J, \lambda>0 .
$$

Concerning the issues in [7], the authors widely use certain estimation: $\mathbb{E}_{\alpha}(z) \leq 1$, $\mathbb{E}_{\alpha, \alpha}(z) \leq \frac{1}{\Gamma(\alpha)}$ for $z \leq 0$, which are reported in [8]. However, concerning our current issues, we need more explicit asymptotic behavior on Mittag-Leffler functions $\mathbb{E}_{\alpha}(z)$ and $\mathbb{E}_{\alpha, \alpha}(z)$ for $z>0$. Following Theorem 2.3 in [11], for $z>0, \mathbb{E}_{\alpha, \alpha}(z)$ can be formulated by

$$
\mathbb{E}_{\alpha, \alpha}(z)=\frac{1}{\alpha} z^{\frac{1-\alpha}{\alpha}} e^{z^{\frac{1}{\alpha}}}+\int_{0}^{\infty} K_{\alpha, \alpha}(r, z) d r
$$

where

$$
K_{\alpha, \alpha}(r, z)=\frac{1}{\pi \alpha} r^{\frac{1-\alpha}{\alpha}} e^{-r^{\frac{1}{\alpha}}} \frac{r \sin (\pi(1-\alpha))}{r^{2}-2 r z \cos (\pi \alpha)+z^{2}} .
$$

It is remarkable that Cong et al. [12] apply the above formula to obtain explicit asymptotic behavior on Mittag-Leffler functions $\mathbb{E}_{\alpha}(z)$ and $\mathbb{E}_{\alpha, \alpha}(z)$ for $z>0$ (see Lemma 2.2). These explicit asymptotic properties are helpful to solve our problems.

In Section 2, we introduce notations, definitions, and preliminary facts. In Section 3, we mainly prove exp-type Ulam-Hyers stability results for equation (2) on a compact interval $[0,1]$. In Section 4 , we mainly prove exp-type Ulam-Hyers stability result for equation (2) on an unbounded interval $(1, \infty)$. Finally, examples are given to illustrate our theoretical results.

\section{Preliminaries}

Let $C(J, \mathbb{R})$ be the Banach space of all continuous functions from $J$ into $\mathbb{R}$ with the $\lambda^{\frac{1}{\alpha}}$ norm $\|y\|_{\lambda \frac{1}{\alpha}}=\sup _{t \in J} e^{-\lambda^{\frac{1}{\alpha}} t}|y(t)|$.

Definition 2.1 (see [13]) The Caputo derivative of order $\gamma$ for a function $f:[0, \infty) \rightarrow \mathbb{R}$ can be written as

$$
{ }^{\mathrm{c}} D_{t}^{\gamma} f(t)={ }^{\mathrm{L}} D_{t}^{\gamma}\left(f(t)-\sum_{k=0}^{n-1} \frac{t^{k}}{k !} f^{(k)}(0)\right), \quad t>0, n-1<\gamma<n,
$$


where ${ }^{\mathrm{L}} D_{t}^{\gamma} f$ denotes the Riemann-Liouville derivative of order $\gamma$ with the lower limit zero for a function $f$, which is given by

$$
{ }^{\mathrm{L}} D_{t}^{\gamma} f(t)=\frac{1}{\Gamma(n-\gamma)} \frac{d^{n}}{d t^{n}} \int_{0}^{t} \frac{f(s)}{(t-s)^{\gamma+1-n}} d s, \quad t>0, n-1<\gamma<n .
$$

We need asymptotic behavior of Mittag-Leffler functions $\mathbb{E}_{\alpha}(z)$ and $\mathbb{E}_{\alpha, \alpha}(z)$ for $z>0$.

Lemma 2.2 (see Lemma 3(i), (ii) and Lemma 5(ii); Lemma 6(ii) via their proofs in [12]) Let $\alpha \in(0,1)$ and $\lambda>0$ be arbitrary.

(i) For all $t>0,\left|\mathbb{E}_{\alpha}\left(\lambda t^{\alpha}\right)-\frac{1}{\alpha} e^{\lambda \frac{1}{\alpha}} t\right| \leq \frac{m(\alpha, \lambda)}{t^{\alpha}}$, where

$$
m(\alpha, \lambda)=\max \left\{\frac{\int_{0}^{\infty} e^{-r^{\frac{1}{\alpha}}} d r}{\sin (\pi \alpha) \pi \alpha \lambda}, \frac{\int_{0}^{\infty} r^{\frac{1}{\alpha}} e^{-r^{\frac{1}{\alpha}}} d r}{\sin (\pi \alpha) \pi \alpha \lambda^{2}}\right\} .
$$

(ii) For all $t>0$,

$$
\left|t^{\alpha-1} \mathbb{E}_{\alpha, \alpha}\left(\lambda t^{\alpha}\right)-\frac{1}{\alpha} \lambda^{\frac{1-\alpha}{\alpha}} e^{\lambda \frac{1}{\alpha} t}\right| \leq \frac{m(\alpha, \lambda)}{t^{\alpha+1}}
$$

(iii) For all $t \in[0,1]$,

$$
\int_{0}^{t}\left|(t-s)^{\alpha-1} \mathbb{E}_{\alpha, \alpha}\left(\lambda(t-s)^{\alpha}\right)-\lambda^{\frac{1-\alpha}{\alpha}} \mathbb{E}_{\alpha}\left(\lambda t^{\alpha}\right) e^{-\lambda \frac{1}{\alpha} s}\right| d s \leq \frac{\mathbb{E}_{\alpha, \alpha}(\lambda)}{\alpha}+\frac{\mathbb{E}_{\alpha}(\lambda)}{\lambda} .
$$

(iv) For all $t \in(1, \infty)$,

$$
\begin{aligned}
& \int_{0}^{t}\left|(t-s)^{\alpha-1} \mathbb{E}_{\alpha, \alpha}\left(\lambda(t-s)^{\alpha}\right)-\lambda^{\frac{1-\alpha}{\alpha}} \mathbb{E}_{\alpha}\left(\lambda t^{\alpha}\right) e^{-\lambda \frac{1}{\alpha}} s\right| d s \\
& \quad \leq m(\alpha, \lambda)\left(\frac{1}{\alpha}+\frac{1}{\lambda}\right)+\mathbb{E}_{\alpha, \alpha+1}(\lambda)+\frac{1}{\alpha} \lambda^{\frac{1-\alpha}{\alpha}} e^{\lambda \frac{1}{\alpha}}+\frac{m(\alpha, \lambda)}{\lambda}:=M(\alpha, \lambda) .
\end{aligned}
$$

Remark 2.3 A function $y \in C(J, \mathbb{R})$ is a solution of inequality (3) if and only if there exists a function $g \in C(J, \mathbb{R})$ (which is not dependent on $y$ ) such that (i) $|g(t)| \leq \epsilon, t \in J$, (ii) ${ }^{\mathrm{c}} D_{t}^{\alpha} y(t)=\lambda y(t)+f(t, y(t))+g(t), t \in J$.

Lemma 2.4 Let $y \in C([0, \infty), \mathbb{R})$ be a solution of inequality (3). Then $y$ is a solution of the following integral inequality:

$$
\begin{aligned}
\mid y(t) & -\mathbb{E}_{\alpha}\left(\lambda t^{\alpha}\right) y(0)-\int_{0}^{t}(t-s)^{\alpha-1} \mathbb{E}_{\alpha, \alpha}\left(\lambda(t-s)^{\alpha}\right) f(s, y(s)) d s \mid \\
\leq & \int_{0}^{t} \frac{m(\alpha, \lambda) \epsilon}{(t-s)^{\alpha+1}} d s+\frac{e^{\lambda \frac{1}{\alpha}} \epsilon}{\alpha \lambda}, \quad t \in[0, \infty) .
\end{aligned}
$$

Proof Indeed, by Remark 2.3, we have

$$
{ }^{\mathrm{c}} D_{t}^{\alpha} y(t)=\lambda y(t)+f(t, y(t))+g(t), \quad t \in[0, \infty) .
$$


From pp.140-141, (3.1.32)-(3.1.34) in [13] we have

$$
\begin{aligned}
y(t)= & \mathbb{E}_{\alpha}\left(\lambda t^{\alpha}\right) y(0)+\int_{0}^{t}(t-s)^{\alpha-1} \mathbb{E}_{\alpha, \alpha}\left(\lambda(t-s)^{\alpha}\right) f(s, y(s)) d s \\
& +\int_{0}^{t}(t-s)^{\alpha-1} \mathbb{E}_{\alpha, \alpha}\left(\lambda(t-s)^{\alpha}\right) g(s) d s, \quad t \in[0, \infty) .
\end{aligned}
$$

For $t=0, y(0)-\mathbb{E}_{\alpha}(0) y(0)=0<\epsilon$, where we use the fact $\mathbb{E}_{\alpha}(0)=1$.

For all $\infty>t>0$, we have

$$
\begin{aligned}
\mid y(t) & -\mathbb{E}_{\alpha}\left(\lambda t^{\alpha}\right) y(0)-\int_{0}^{t}(t-s)^{\alpha-1} \mathbb{E}_{\alpha, \alpha}\left(\lambda(t-s)^{\alpha}\right) f(s, y(s)) d s \mid \\
= & \left|\int_{0}^{t}(t-s)^{\alpha-1} \mathbb{E}_{\alpha, \alpha}\left(\lambda(t-s)^{\alpha}\right) g(s) d s\right| \\
\leq & \int_{0}^{t}\left|(t-s)^{\alpha-1} \mathbb{E}_{\alpha, \alpha}\left(\lambda(t-s)^{\alpha}\right)-\frac{1}{\alpha} \lambda^{\frac{1-\alpha}{\alpha}} e^{\lambda \frac{1}{\alpha}(t-s)}\right||g(s)| d s \\
& +\int_{0}^{t}\left|\frac{1}{\alpha} \lambda^{\frac{1-\alpha}{\alpha}} e^{\lambda \frac{1}{\alpha}(t-s)}\right||g(s)| d s \\
\leq & \epsilon \int_{0}^{t} \frac{m(\alpha, \lambda)}{(t-s)^{\alpha+1}} d s+\epsilon \int_{0}^{t} \frac{1}{\alpha} \lambda^{\frac{1-\alpha}{\alpha}} e^{\lambda \frac{1}{\alpha}(t-s)} d s \\
= & \int_{0}^{t} \frac{m(\alpha, \lambda) \epsilon}{(t-s)^{\alpha+1}} d s+\frac{e^{\lambda \frac{1}{\alpha} t} \epsilon}{\alpha \lambda}-\frac{\epsilon}{\alpha \lambda} \\
\leq & \int_{0}^{t} \frac{m(\alpha, \lambda) \epsilon}{(t-s)^{\alpha+1}} d s+\frac{e^{\lambda \frac{1}{\alpha}} t \epsilon}{\alpha \lambda},
\end{aligned}
$$

where we use Lemma 2.2(ii). The decried result is obtained.

Remark 2.5 Note that for some arbitrary fixed $t_{0}>0$ and $t_{0}>\delta>0$,

$$
\int_{0}^{t_{0}} \frac{1}{\left(t_{0}-s\right)^{\alpha+1}} d s=\lim _{\delta \rightarrow 0} \int_{0}^{t_{0}-\delta} \frac{1}{\left(t_{0}-s\right)^{\alpha+1}} d s=\lim _{\delta \rightarrow 0} \frac{1}{\alpha}\left(\delta^{-\alpha}-t_{0}^{-\alpha}\right)=\infty
$$

So it is not possible to obtain some explicit estimation in this case.

Next, we divide our time interval $[0, \infty)$ into two subintervals $[0,1]$ and $(1, \infty)$.

Remark 2.6 Let $y \in C([0,1], \mathbb{R})$ be a solution of inequality (3). Then $y$ is a solution of the following integral inequality:

$$
\begin{aligned}
\mid y(t) & -\mathbb{E}_{\alpha}\left(\lambda t^{\alpha}\right) y(0)-\int_{0}^{t}(t-s)^{\alpha-1} \mathbb{E}_{\alpha, \alpha}\left(\lambda(t-s)^{\alpha}\right) f(s, y(s)) d s \mid \\
\leq & \int_{0}^{t}\left|(t-s)^{\alpha-1} \mathbb{E}_{\alpha, \alpha}\left(\lambda(t-s)^{\alpha}\right)-\lambda^{\frac{1-\alpha}{\alpha}} \mathbb{E}_{\alpha}\left(\lambda t^{\alpha}\right) e^{-\lambda^{\frac{1}{\alpha}}} s\right||g(s)| d s \\
& +\int_{0}^{t}\left|\lambda \frac{1-\alpha}{\alpha} \mathbb{E}_{\alpha}\left(\lambda t^{\alpha}\right) e^{-\lambda \frac{1}{\alpha}} s\right||g(s)| d s \\
\leq & \epsilon\left(\frac{\mathbb{E}_{\alpha, \alpha}(\lambda)}{\alpha}+\frac{\mathbb{E}_{\alpha}(\lambda)}{\lambda}\right)+\epsilon \int_{0}^{t} \lambda^{\frac{1-\alpha}{\alpha}} \mathbb{E}_{\alpha}\left(\lambda t^{\alpha}\right) e^{-\lambda^{\frac{1}{\alpha}} s} d s
\end{aligned}
$$




$$
\begin{aligned}
& \leq \epsilon\left(\frac{\mathbb{E}_{\alpha, \alpha}(\lambda)}{\alpha}+\frac{\mathbb{E}_{\alpha}(\lambda)}{\lambda}\right)+\epsilon \frac{\mathbb{E}_{\alpha}(\lambda)}{\lambda}\left(1-e^{-\lambda} \frac{1}{\alpha} t\right) \\
& \leq \epsilon\left(\frac{\mathbb{E}_{\alpha, \alpha}(\lambda)}{\alpha}+\frac{2 \mathbb{E}_{\alpha}(\lambda)}{\lambda}\right), \quad t \in[0,1],
\end{aligned}
$$

where we use Remark 2.3, Lemma 2.2(iii) and the fact that $\mathbb{E}_{\alpha}$ is an increasing function.

Remark 2.7 Let $y \in C((1, \infty), \mathbb{R})$ be a solution of inequality (3). Then $y$ is a solution of the following integral inequality:

$$
\begin{aligned}
\mid y(t) & -\mathbb{E}_{\alpha}\left(\lambda t^{\alpha}\right) y(0)-\int_{0}^{t}(t-s)^{\alpha-1} \mathbb{E}_{\alpha, \alpha}\left(\lambda(t-s)^{\alpha}\right) f(s, y(s)) d s \mid \\
\leq & \int_{0}^{t}\left|(t-s)^{\alpha-1} \mathbb{E}_{\alpha, \alpha}\left(\lambda(t-s)^{\alpha}\right)-\lambda \frac{1-\alpha}{\alpha} \mathbb{E}_{\alpha}\left(\lambda t^{\alpha}\right) e^{-\lambda \frac{1}{\alpha}} s\right||g(s)| d s \\
& +\int_{0}^{t}\left|\lambda \frac{1-\alpha}{\alpha} \mathbb{E}_{\alpha}\left(\lambda t^{\alpha}\right) e^{-\lambda \frac{1}{\alpha}} s\right||g(s)| d s \\
\leq & \epsilon M(\alpha, \lambda)+\epsilon \int_{0}^{t} \lambda^{\frac{1-\alpha}{\alpha}} \mathbb{E}_{\alpha}\left(\lambda t^{\alpha}\right) e^{-\lambda \frac{1}{\alpha}} s d s \\
\leq & \epsilon M(\alpha, \lambda)+\epsilon\left(\frac{1}{\lambda \alpha} e^{\lambda \frac{1}{\alpha}} t+\frac{m(\alpha, \lambda)}{\lambda t^{\alpha}}\right), \quad t \in(1, \infty),
\end{aligned}
$$

where we use Remark 2.3, Lemma 2.2(iv) and apply Lemma 2.2(i) to derive the following fact:

$$
\begin{aligned}
\int_{0}^{t} \lambda \lambda^{\frac{1-\alpha}{\alpha}} \mathbb{E}_{\alpha}\left(\lambda t^{\alpha}\right) e^{-\lambda \frac{1}{\alpha} s} d s & =\lambda^{-1} \mathbb{E}_{\alpha}\left(\lambda t^{\alpha}\right)\left(1-e^{-\lambda \frac{1}{\alpha}} t\right) \\
& \leq \lambda^{-1}\left(\frac{1}{\alpha} e^{\lambda \frac{1}{\alpha}} t+\frac{m(\alpha, \lambda)}{t^{\alpha}}\right)\left(1-e^{-\lambda \frac{1}{\alpha} t}\right) \\
& \leq \frac{1}{\lambda \alpha} e^{\lambda \frac{1}{\alpha} t}+\frac{m(\alpha, \lambda)}{\lambda t^{\alpha}}
\end{aligned}
$$

\section{Exp-type Ulam-Hyers stability results on $J=[0,1]$}

Next, we introduce the following assumptions:

$\left(\mathrm{H}_{1}\right) f: J \times \mathbb{R} \rightarrow \mathbb{R}$ is jointly continuous.

$\left(\mathrm{H}_{2}\right)$ There exists $L>0$ such that

$$
|f(t, x)-f(t, y)| \leq L|x-y| \quad \text { for each } t \in J \text { and all } x, y \in \mathbb{R}
$$

$\left(\mathrm{H}_{3}\right) \quad \omega^{\prime}:=1-L\left(\frac{\mathbb{E}_{\alpha, \alpha}(\lambda)}{\alpha}+\frac{\mathbb{E}_{\alpha}(\lambda)}{\lambda}\right)-L \lambda \frac{1-\alpha}{\alpha} \mathbb{E}_{\alpha}(\lambda)>0$.

Theorem 3.1 Assume that $\left(\mathrm{H}_{1}\right),\left(\mathrm{H}_{2}\right)$, and $\left(\mathrm{H}_{3}\right)$ are satisfied. Then equation (2) is exp-type Ulam-Hyers stable on $J=[0,1]$.

Proof Let $y \in C(J, \mathbb{R})$ be a solution of inequality (3). Denote by $x$ the unique solution of the Cauchy problem

$$
\left\{\begin{array}{l}
{ }^{\mathrm{c}} D_{t}^{\alpha} x(t)=\lambda x(t)+f(t, x(t)), \quad t \in J \\
x(0)=y(0)
\end{array}\right.
$$


that is,

$$
x(t)=\mathbb{E}_{\alpha}\left(\lambda t^{\alpha}\right) y(0)+\int_{0}^{t}(t-s)^{\alpha-1} \mathbb{E}_{\alpha, \alpha}\left(\lambda(t-s)^{\alpha}\right) f(s, x(s)) d s .
$$

By using Lemma 2.2(iii) and (5), we have

$$
\begin{aligned}
\mid y(t) & -x(t) \mid \\
\leq & \left|y(t)-\mathbb{E}_{\alpha}\left(\lambda t^{\alpha}\right) y(0)-\int_{0}^{t}(t-s)^{\alpha-1} \mathbb{E}_{\alpha, \alpha}\left(\lambda(t-s)^{\alpha}\right) f(s, x(s)) d s\right| \\
\leq & \left|y(t)-\mathbb{E}_{\alpha}\left(\lambda t^{\alpha}\right) y(0)-\int_{0}^{t}(t-s)^{\alpha-1} \mathbb{E}_{\alpha, \alpha}\left(\lambda(t-s)^{\alpha}\right) f(s, y(s)) d s\right| \\
& +\mid \int_{0}^{t}(t-s)^{\alpha-1} \mathbb{E}_{\alpha, \alpha}\left(\lambda(t-s)^{\alpha}\right) f(s, y(s)) d s \\
& -\int_{0}^{t}(t-s)^{\alpha-1} \mathbb{E}_{\alpha, \alpha}\left(\lambda(t-s)^{\alpha}\right) f(s, x(s)) d s \mid \\
\leq & \epsilon\left(\frac{\mathbb{E}_{\alpha, \alpha}(\lambda)}{\alpha}+\frac{2 \mathbb{E}_{\alpha}(\lambda)}{\lambda}\right) \\
& +L \int_{0}^{t}\left|(t-s)^{\alpha-1} \mathbb{E}_{\alpha, \alpha}\left(\lambda(t-s)^{\alpha}\right)-\lambda \frac{1-\alpha}{\alpha} \mathbb{E}_{\alpha}\left(\lambda t^{\alpha}\right) e^{-\lambda \frac{1}{\alpha} s}\right||y(s)-x(s)| d s \\
& +L \int_{0}^{t}\left|\lambda \frac{1-\alpha}{\alpha} \mathbb{E}_{\alpha}\left(\lambda t^{\alpha}\right) e^{-\lambda \frac{1}{\alpha} s}\right||y(s)-x(s)| d s \\
\leq & \epsilon\left(\frac{\mathbb{E}_{\alpha, \alpha}(\lambda)}{\alpha}+\frac{2 \mathbb{E}_{\alpha}(\lambda)}{\lambda}\right)+L\left(\frac{\mathbb{E}_{\alpha, \alpha}(\lambda)}{\alpha}+\frac{\mathbb{E}_{\alpha}(\lambda)}{\lambda}\right) e^{\lambda \frac{1}{\alpha}}\|x-y\|_{\lambda \frac{1}{\alpha}} \\
& +L t \lambda^{\frac{1-\alpha}{\alpha}} \mathbb{E}_{\alpha}\left(\lambda t^{\alpha}\right)\|x-y\|_{\lambda \lambda^{\frac{1}{\alpha}}} \\
\leq & \epsilon\left(\frac{\mathbb{E}_{\alpha, \alpha}(\lambda)}{\alpha}+\frac{2 \mathbb{E}_{\alpha}(\lambda)}{\lambda}\right)+L e^{\lambda \frac{1}{\alpha}} t\left(\frac{\mathbb{E}_{\alpha, \alpha}(\lambda)}{\alpha}+\frac{\mathbb{E}_{\alpha}(\lambda)}{\lambda}\right)\|x-y\|_{\lambda \frac{1}{\alpha}} \\
& +L \lambda^{\frac{1-\alpha}{\alpha}} \mathbb{E}_{\alpha}(\lambda)\|x-y\|_{\lambda^{\frac{1}{\alpha}}},
\end{aligned}
$$

which yields that

$$
\begin{aligned}
\|x-y\|_{\lambda \frac{1}{\alpha}} \leq & \epsilon\left(\frac{\mathbb{E}_{\alpha, \alpha}(\lambda)}{\alpha}+\frac{2 \mathbb{E}_{\alpha}(\lambda)}{\lambda}\right)+L\left(\frac{\mathbb{E}_{\alpha, \alpha}(\lambda)}{\alpha}+\frac{\mathbb{E}_{\alpha}(\lambda)}{\lambda}\right)\|x-y\|_{\lambda \frac{1}{\alpha}} \\
& +L \lambda \frac{1-\alpha}{\alpha} \mathbb{E}_{\alpha}(\lambda)\|x-y\|_{\lambda^{\frac{1}{\alpha}}} .
\end{aligned}
$$

Thus,

$$
\omega^{\prime}\|x-y\|_{\lambda \frac{1}{\alpha}} \leq \epsilon\left(\frac{\mathbb{E}_{\alpha, \alpha}(\lambda)}{\alpha}+\frac{2 \mathbb{E}_{\alpha}(\lambda)}{\lambda}\right) .
$$

This yields that

$$
|y(t)-x(t)| \leq \frac{\epsilon}{\omega^{\prime}}\left(\frac{\mathbb{E}_{\alpha, \alpha}(\lambda)}{\alpha}+\frac{2 \mathbb{E}_{\alpha}(\lambda)}{\lambda}\right) e^{\lambda \frac{1}{\alpha} t}, \quad t \in J .
$$

The proof is completed. 
Remark 3.2 Under the assumptions of Theorem 3.1, consider

$$
\left\{\begin{array}{l}
{ }^{\mathrm{c}} D_{t}^{\alpha} x(t)=\lambda x(t)+f(t, x(t)), \quad t \in J, \lambda>0, \\
x(0)=x_{0} .
\end{array}\right.
$$

Define $P: C(J, \mathbb{R}) \rightarrow C(J, \mathbb{R})$ as follows:

$$
(P x)(t)=\mathbb{E}_{\alpha}\left(\lambda t^{\alpha}\right) x_{0}+\int_{0}^{t}(t-s)^{\alpha-1} \mathbb{E}_{\alpha, \alpha}\left(\lambda(t-s)^{\alpha}\right) f(s, x(s)) d s
$$

Then take $t \in J$ and $x, y \in C(J, \mathbb{R})$, one can obtain

$$
\|P x-P y\|_{\lambda^{\frac{1}{\alpha}}} \leq\left(1-\omega^{\prime}\right)\|x-y\|_{\lambda^{\frac{1}{\alpha}}}<\|x-y\|_{\lambda^{\frac{1}{\alpha}}} .
$$

In view of the contraction mapping principle, that $P$ has a unique fixed point. Thus, problem (7) has a unique solution.

\section{Exp-type Ulam-Hyers stability result on $J=(1, \infty)$}

Denote $L^{1}\left([0, \infty), \mathbb{R}^{+}\right)$by all integrable functions from $[0, \infty)$ into $\mathbb{R}^{+}$. Denote $C^{\infty}([0, \infty)$, $\mathbb{R}^{+}$) by all continuous functions from $[0, \infty)$ into $\mathbb{R}^{+}$with $\|y\|_{\infty}=\sup _{t \in[0, \infty)}|y(t)|$.

Next, we introduce the following assumptions:

$\left(\mathrm{H}_{2}^{\prime}\right)$ There exists $L(\cdot) \in L^{1}\left([0, \infty), \mathbb{R}^{+}\right) \cap C^{\infty}\left([0, \infty), \mathbb{R}^{+}\right)$such that

$$
|f(t, x)-f(t, y)| \leq L(t)|x-y| \quad \text { for each } t \in J \text { and all } x, y \in \mathbb{R} \text {. }
$$

$\left(\mathrm{H}_{3}^{\prime}\right)$ Set $\|L\|_{\infty}=\sup _{t \in[0, \infty)}|L(t)|$ and

$$
k_{1}=\sup _{t \in[0, \infty)} \int_{0}^{t} L(s) d s=\int_{0}^{\infty} L(s) d s<\infty
$$

such that $\omega^{\prime \prime}=1-\|L\|_{\infty} M(\alpha, \lambda)-k_{1} \lambda^{\frac{1-\alpha}{\alpha}} \frac{1}{\alpha}>0$.

Theorem 4.1 Assume that $\left(\mathrm{H}_{1}\right),\left(\mathrm{H}_{2}^{\prime}\right)$, and $\left(\mathrm{H}_{3}^{\prime}\right)$ are satisfied. Then equation (2) is exp-type Ulam-Hyers stable on $J=(1, \infty)$.

Proof To achieve our aim, we apply Lemma 2.2(i), (vi), and (6) to obtain

$$
\begin{aligned}
\mid y(t) & -x(t) \mid \\
\leq & \epsilon M(\alpha, \lambda)+\epsilon\left(\frac{1}{\lambda \alpha} e^{\lambda \frac{1}{\alpha}} t+\frac{m(\alpha, \lambda)}{\lambda t^{\alpha}}\right) \\
& +\|L\|_{\infty} \int_{0}^{t}\left|(t-s)^{\alpha-1} \mathbb{E}_{\alpha, \alpha}\left(\lambda(t-s)^{\alpha}\right)-\lambda \frac{1-\alpha}{\alpha} \mathbb{E}_{\alpha}\left(\lambda t^{\alpha}\right) e^{-\lambda \frac{1}{\alpha}} s\right||y(s)-x(s)| d s \\
& +\int_{0}^{t} L(s)\left|\lambda^{\frac{1-\alpha}{\alpha}} \mathbb{E}_{\alpha}\left(\lambda t^{\alpha}\right) e^{-\lambda \frac{1}{\alpha}} s\right||y(s)-x(s)| d s \\
\leq & \epsilon M(\alpha, \lambda)+\epsilon\left(\frac{1}{\lambda \alpha} e^{\lambda \frac{1}{\alpha}} t+\frac{m(\alpha, \lambda)}{\lambda t^{\alpha}}\right)
\end{aligned}
$$




$$
\begin{aligned}
& +\|L\|_{\infty} e^{\lambda^{\frac{1}{\alpha}}} M(\alpha, \lambda)\|x-y\|_{\lambda^{\frac{1}{\alpha}}}+\int_{0}^{\infty} L(s) d s \lambda^{\frac{1-\alpha}{\alpha}}\left(\frac{1}{\alpha} e^{\lambda^{\frac{1}{\alpha}} t}+\frac{m(\alpha, \lambda)}{t^{\alpha}}\right)\|x-y\|_{\lambda^{\frac{1}{\alpha}}} \\
\leq & \epsilon M(\alpha, \lambda)+\epsilon\left(\frac{1}{\lambda \alpha} e^{\lambda \frac{1}{\alpha} t}+\frac{m(\alpha, \lambda)}{\lambda t^{\alpha}}\right) \\
& +\|L\|_{\infty} e^{\lambda \frac{1}{\alpha} t} M(\alpha, \lambda)\|x-y\|_{\lambda^{\frac{1}{\alpha}}}+k_{1} \lambda^{\frac{1-\alpha}{\alpha}}\left(\frac{1}{\alpha} e^{\lambda \frac{1}{\alpha} t}+\frac{m(\alpha, \lambda)}{t^{\alpha}}\right)\|x-y\|_{\lambda^{\frac{1}{\alpha}}},
\end{aligned}
$$

which yields that

$$
\|x-y\|_{\lambda^{\frac{1}{\alpha}}} \leq \epsilon \frac{1}{\lambda \alpha}+\left(\|L\|_{\infty} M(\alpha, \lambda)+k_{1} \lambda^{\frac{1-\alpha}{\alpha}} \frac{1}{\alpha}\right)\|x-y\|_{\lambda^{\frac{1}{\alpha}}} .
$$

This implies that

$$
\omega^{\prime \prime}\|x-y\|_{\lambda \frac{1}{\alpha}} \leq \frac{\epsilon}{\alpha \lambda} .
$$

Finally,

$$
|y(t)-x(t)| \leq \frac{1}{\alpha \lambda \omega^{\prime \prime}} \epsilon e^{\lambda \frac{1}{\alpha} t}, \quad t \in J .
$$

Thus, the proof is finished.

Remark 4.2 Under the assumptions of Theorem 4.1, problem (7) also has a unique solution. The uniqueness result can be shown in a similar way as in Remark 3.2. So we omit the details here.

\section{Examples}

Now we give some examples to illustrate our theoretical results.

Example 5.1 Let $\alpha=\frac{1}{2}$ and $\lambda=\frac{1}{4}$. We consider the fractional order differential equation

$$
{ }^{\mathrm{c}} D_{t}^{\frac{1}{2}} x(t)=\frac{1}{4} x(t)+\frac{1}{l} \sin x(t), \quad t \in[0,1], l>0,
$$

and the inequality

$$
\left|{ }^{\mathrm{c}} D_{t}^{\frac{1}{2}} y(t)-\frac{1}{4} y(t)-\frac{1}{l} \sin y(t)\right| \leq \epsilon, \quad t \in[0,1] .
$$

Let $y \in C([0,1], \mathbb{R})$ be a solution of inequality (9). Then there exists a function $g(t)=$ $\epsilon e^{t-1} \in C([0,1], \mathbb{R})$ such that $|g(t)| \leq \epsilon, t \in[0,1]$, and ${ }^{\mathrm{c}} D_{t}^{\frac{1}{2}} y(t)=\frac{1}{4} y(t)+\frac{1}{l} \sin y(t)+g(t), t \in$ $[0,1]$.

Define $f(t, x(t))=\frac{1}{l} \sin x(t)$ for $t \in[0,1]$ and $\operatorname{set} L=\frac{1}{l}$. Then $\left(\mathrm{H}_{1}\right)$ and $\left(\mathrm{H}_{2}\right)$ hold. Moreover, we choose $l=2\left[\left(2 \mathbb{E}_{\frac{1}{2}, \frac{1}{2}}\left(\frac{1}{4}\right)+4 \mathbb{E}_{\frac{1}{2}}\left(\frac{1}{4}\right)\right)+\frac{1}{4} \mathbb{E}_{\frac{1}{2}}\left(\frac{1}{4}\right)\right]$, then

$$
\omega^{\prime}:=1-\frac{1}{l}\left[\left(2 \mathbb{E}_{\frac{1}{2}, \frac{1}{2}}\left(\frac{1}{4}\right)+4 \mathbb{E}_{\frac{1}{2}}\left(\frac{1}{4}\right)\right)+\frac{1}{4} \mathbb{E}_{\frac{1}{2}}\left(\frac{1}{4}\right)\right]=\frac{1}{2}>0,
$$

which implies that $\left(\mathrm{H}_{3}\right)$ holds. 
By Theorem 3.1, we have

$$
|y(t)-x(t)| \leq 2\left(2 \mathbb{E}_{\frac{1}{2}, \frac{1}{2}}\left(\frac{1}{4}\right)+8 \mathbb{E}_{\frac{1}{2}}\left(\frac{1}{4}\right)\right) \epsilon e^{\frac{1}{16} t}, \quad t \in[0,1] .
$$

Thus, equation (8) is exp-type Ulam-Hyers stable on $[0,1]$ with $c=4\left(\mathbb{E}_{\frac{1}{2}, \frac{1}{2}}\left(\frac{1}{4}\right)+4 \mathbb{E}_{\frac{1}{2}}\left(\frac{1}{4}\right)\right)$.

Example 5.2 Let $\alpha=\frac{1}{2}, \lambda=1$. We consider the fractional order differential equation

$$
{ }^{\mathrm{c}} D_{t}^{\frac{1}{2}} x(t)=x(t)+\frac{1}{l e^{t}} \frac{|x(t)|}{1+|x(t)|}, \quad t \in(1, \infty), l>0,
$$

and the inequality

$$
\left|{ }^{\mathrm{c}} D_{t}^{\frac{1}{2}} y(t)-y(t)-\frac{1}{l e^{t}} \frac{|y(t)|}{1+|y(t)|}\right| \leq \epsilon e^{t}, \quad t \in(1, \infty) .
$$

Let $y$ be a solution of (11). Then there exists a function $g(t)=\epsilon e^{1-t} \in C((1, \infty), \mathbb{R})$ such that $|g(t)| \leq \epsilon, t \in[0,1]$, and ${ }^{\mathrm{c}} D_{t}^{\frac{1}{2}} y(t)=y(t)+\frac{1}{l e^{t}} \frac{|y(t)|}{1+|y(t)|}+g(t), t \in(1, \infty)$.

Define $f(t, x(t))=\frac{1}{l e^{t}} \frac{|x(t)|}{1+|x(t)|}$ for $t \in(1, \infty)$ and set $L(t)=\frac{1}{l e^{t}}, t \in(1, \infty)$. Then $\left(\mathrm{H}_{1}\right)$ and $\left(\mathrm{H}_{2}^{\prime}\right)$ hold. Moreover, $\|L\|_{\infty}=\frac{1}{l}$ and $k_{1}=\int_{0}^{\infty} L(s) d s=\frac{1}{l}<\infty$. Now, choose $l=2\left(M\left(\frac{1}{2}, 1\right)+2\right)$, where $M\left(\frac{1}{2}, 1\right)=\frac{4}{\sqrt{\pi}}+\mathbb{E}_{\frac{1}{2}, \frac{3}{2}}(1)+2 e$. Then $\omega^{\prime \prime}=1-\frac{M\left(\frac{1}{2}, 1\right)}{l}-\frac{2}{l}=\frac{1}{2}$, which implies that $\left(\mathrm{H}_{3}^{\prime}\right)$ holds.

By Theorem 4.1, we have

$$
|y(t)-x(t)| \leq 4 \epsilon e^{t}, \quad t \in(1, \infty)
$$

Thus, equation (10) is exp-type Ulam-Hyers stable on $(1, \infty)$ with $c=4$.

\section{Competing interests}

The authors declare that they have no competing interests.

\section{Authors' contributions}

This work was carried out in collaboration between all authors. ZG, XY, and JRW proved the theorems, interpreted the results, and wrote the article. All authors defined the research theme, read and approved the manuscript.

\section{Author details}

${ }^{1}$ College of Applied Mathematics, Shanxi University of Finance and Economics, Taiyuan, Shanxi 030006, P.R. China.

${ }^{2}$ Department of Mathematics, Guizhou University, Guiyang, Guizhou 550025, P.R. China.

\section{Acknowledgements}

The authors are grateful to the referees for their careful reading of the manuscript and valuable comments. The authors thank for the help from the editor too. This work is partially supported by the Youth Science Foundation of Shanxi University of Finance and Economic (2014003) and National Natural Science Foundation of China (11201091).

Received: 2 March 2015 Accepted: 18 July 2015 Published online: 04 August 2015

\section{References}

1. Jung, S-M: Hyers-Ulam-Rassias Stability of Functional Equations in Mathematical Analysis. Hadronic Press, Palm Harbor (2001)

2. Jung, S-M: Hyers-Ulam-Rassias Stability of Functional Equations in Nonlinear Analysis. Springer, New York (2011)

3. Brzdęk, J, Brillouët-Belluot, N, Ciepliński, K, Xu, B: Ulam's type stability. Abstr. Appl. Anal. 2012, Article ID 329702 (2012)

4. Rus, IA: Ulam stability of ordinary differential equations. Stud. Univ. Babeş-Bolyai, Math. 54, 125-133 (2009)

5. Popa, D, Raşa, I: On the Hyers-Ulam stability of the linear differential equation. J. Math. Anal. Appl. 381, $530-537$ (2011)

6. Hegyi, B, Jung, S-M: On the stability of Laplace's equation. Appl. Math. Lett. 26, 549-552 (2013)

7. Wang, J, Li, X: $\mathbb{E}_{\alpha}$-Ulam type stability of fractional order ordinary differential equations. J. Appl. Math. Comput. 45, 449-459 (2014) 
8. Wang, J, Fečkan, M, Zhou, Y: Presentation of solutions of impulsive fractional Langevin equations and existence results. Eur. Phys. J. Spec. Top. 222, 1855-1872 (2013)

9. Ye, H, Gao, J, Ding, Y: A generalized Gronwall inequality and its application to a fractional differential equation. J. Math. Anal. Appl. 328, 1075-1081 (2007)

10. Hyers, DH: On the stability of the linear functional equation. Proc. Natl. Acad. Sci. USA 27, 222-224 (1941)

11. Gorenflo, R, Loutchko, J, Luchko, Y: Computation of the Mittag-Leffler function $E_{\alpha \beta}(z)$ and its derivative. Fract. Calc. Appl. Anal. 5, $491-518$ (2002). Correction: Fract. Calc. Appl. Anal. 6, 111-112 (2003)

12. Cong, ND, Doan, TS, Siegmund, S, Tuan, HT: On stable manifolds for planar fractional differential equations. Appl. Math. Comput. 226, 157-168 (2014)

13. Kilbas, AA, Srivastava, HM, Trujillo, Jj: Theory and Applications of Fractional Differential Equations. Elsevier, Amsterdam (2006)

Submit your manuscript to a SpringerOpen ${ }^{\odot}$ journal and benefit from:

- Convenient online submission

Rigorous peer review

- Immediate publication on acceptance

- Open access: articles freely available online

- High visibility within the field

- Retaining the copyright to your article 